\title{
Nonlinear Koopman Modes and a Precursor to Power System Swing Instabilities
}

\author{
$\operatorname{AUTHOR}(\mathrm{S})$ :
}

Susuki, Yoshihiko; Mezic, Igor

\section{CITATION:}

Susuki, Yoshihiko ... [et al]. Nonlinear Koopman Modes and a Precursor to Power System Swing Instabilities. IEEE Transactions on Power Systems 2012, 27(3): 1182-1191

\section{ISSUE DATE:}

2012-08

URL:

http://hdl.handle.net/2433/178794

\section{RIGHT:}

(c) 2012 IEEE. Personal use of this material is permitted. Permission from IEEE must be obtained for all other uses, in any current or future media, including reprinting/republishing this material for advertising or promotional purposes,

creating new collective works, for resale or redistribution to servers or lists, or reuse of any copyrighted component of this work in other works.; This is not the published version. Please cite only the published version.; この論文は出版社版

でありません。引用の際には出版社版をご確認ご利用ください。 


\title{
Nonlinear Koopman Modes and a Precursor to Power System Swing Instabilities
}

\author{
Yoshihiko Susuki, Member, Igor Mezić, Member
}

\begin{abstract}
We suggest a precursor to phenomena of loss of transient stability in multi-machine power systems. This precursor is based on discovery of [Y. Susuki, I. Mezić, and T. Hikihara, J. Nonlinear Sci., vol. 21, no. 3, pp. 403-439, June 2011.], an emergent transmission path of energy from many oscillatory modes to one oscillatory mode that represents an instability phenomenon of interest. The pathway from high frequency modes to the lowest frequency mode is called the Coherent Swing Instability (CSI). The modes are extracted from sensor data or data provided by simulation outputs of power system oscillations by using the Koopman Mode Analysis that is based on a fully nonlinear spectral theory and represents an extension of linear oscillatory mode analysis. The CSI transmission path is identified by computation of the so-called action transfer operator that is derived by refining a mathematical model of transient stability using the Koopman Mode Analysis. This provides a new technique for monitoring the loss of transient stability by a combination of practical data, mathematical modeling, and computation.
\end{abstract}

Index Terms-power system, transient stability, precursor to instability, Koopman mode, energy transfer, coherent swing instability

\section{INTRODUCTION}

W E STUDY PRECURSORS to power system dynamic phenomena. The notion of precursor used here is a diagnostic phenomenon that can occur before the dynamic phenomenon of interest emerges in power systems. Our purpose is to identify a precursor to instabilities in short-term swing dynamics by using numerical simulations (possibly, data sampled in practice) and mathematical models, so that system operator can monitor, analyze, and control the phenomena of interest.

Monitoring dynamic phenomena of the power system has received a considerable attention in recent years: see e.g. [1]. The so-called Smart Grid vision aims to synthesize the next architecture of power system that operates in an energy-efficient way, handles renewable energy sources, and archives robust electricity supply against cyber-security attacks and physical instabilities [2]. Examples of the physical instabilities include

Manuscript received November 21, 2010; revised May 3 and November 4, 2011; accepted December 19, 2011. This work was supported in part by JSPS Postdoctoral Fellowships for Research Abroad and in part NICT Research Project ICE-IT (Integrated Technology of Information, Communications, and Energy).

Y. Susuki is with the Department of Electrical Engineering, Kyoto University, Nishikyo, Kyoto 615-8510 Japan (e-mail: susuki@ieee.org). During parts of the work in this paper, he was at the Department of Mechanical Engineering, University of California, Santa Barbara, CA 93106-5370 United States.

I. Mezić is with the Department of Mechanical Engineering, University of California, Santa Barbara, CA 93106-5370 United States (e-mail: mezic@engineering.ucsb.edu). the U.S. and Canada blackout on August 14, 2003 [3]. It has been widely accepted that the lack of accuracy of measurement of power system (dynamic) states is one important cause of such blackouts of the existing power system. Monitoring dynamic phenomena, especially instabilities, is an essential step in developing tools necessary for analysis and control of the next power system. In this paper, as a dynamical basis of monitoring process, we study a precursor to instabilities in short-term coupled swing dynamics.

Related Work.--In [4], [5], the authors explained a mechanism of long-term swing instability as an internal resonance of two oscillatory modes coupled with system nonlinearity. The authors use the classical notion of linear modes and derive a reduced order model that represents the envelope of long-term swing such as inter-area one. In [6] the authors considered how changes in power system parameters could cause low frequency inter-area oscillations. Consider two damped oscillatory modes for a system of linearized power system models. In an ideal situation the two linear modes coincide exactly: the coincidence is called a 1:1 resonance. If the linearization is not diagonalizable at the resonance, the resonance is called a strong resonance. The authors show the strong modal resonance is a precursor to the oscillatory instability. In [7] the authors considered a signal-based approach for real-time estimation of the distance to instability. Based on the notion of noisy precursors of bifurcations [8], the authors adopt, as precursors, impending instability features of power spectrum density of a measured output that are obtained by excitation of additive white Gaussian noise. Optimal output for identifying the precursors is determined by the input-to-state participation factors for linear systems.

Contributions.-The main contribution of this paper is to identify a precursor to phenomena in loss of short-term swing stability, the so-called transient stability in multi-machine power systems. Loss of transient stability is associated with non-local dynamics of a power system, in the sense that it does not happen upon an infinitesimally small perturbation around an equilibrium of the system (see Sec. II for definition). It compasses the situation when the system escapes a predefined set around the equilibrium. In this way the loss of transient stability is nonlocal. A technique taking into nonlinear natures of the phenomenon is required for identifying precursors to such nonlocal phenomena. In this paper, we employ the Koopman Mode (KM) that is a nonlinear extension of linear oscillatory mode and is introduced in [9]-[11]. Koopman pioneered the use of linear transformations on Hilbert space to analyze (nonlinear) Hamiltonian systems by introducing the so-called Koopman operator and studying its spectrum 
[12]. This linear, infinite-dimensional operator is defined for any nonlinear dynamical systems [13], [14]. Even if the governing dynamics of a system are finite-dimensional, the Koopman operator is infinite-dimensional and does not rely on linearization: indeed, it captures the full information of the nonlinear dynamical system. In [15] the authors identified a relationship between generalized Fourier analysis [16] and eigen-functions of the Koopman operator. In [9] the author showed via spectral analysis of the Koopman operator that single-frequency modes can be embedded in highly nonlinear, spatiotemporal dynamics. These modes are later named the KMs [10]. In this reference, the authors provide an algorithm for computing KMs from numerical and experimental data. In this paper, we show that the KM Analysis provides a precursor to one type of loss of transient stability, Coherent Swing Instability (CSI) [17], [18]. CSI is an emergent and undesirable phenomenon of synchronous machines in a power system, in which a group of machines in the system coherently loses synchronism with the rest of the system after being subjected to a finite, possibly local disturbance. The dynamical mechanism of CSI explains how local plant mode oscillations, inter-area mode instability, and multi-swing instability interact to destabilize a power system. The full analysis of CSI is presented in [18]. The precursor which we identify in this paper is an emergent transmission path of energy from high KMs to the lowest KM with flat basis vector, which we term the coherent KM (see Sec. V). The lowest mode corresponds to the collective mode described by the well-known Center-OfAngle (COA) variables. This result is consistent with that in [19] that analyzes dynamics of high-dimensional oscillatory systems with strong local linear interconnections and weak long-range nonlinear forces. The current result is more general, because it is obtained for a set of KMs embedded in dynamics of a high-dimensional oscillatory system with nonlinear local interconnections. In this paper, we develop analysis tools that enable us to derive mathematical models relevant to analysis of instability phenomena (see Sec. IV). The tools include 1) model reduction based on the KM and Petrov-Galerkin projective approximation, and 2) a linear, finite-dimensional operator quantifying a transmission path of energy between different KMs, to which we refer as the action transfer operator. Their development is the other contribution of this paper.

Note that the precursor identified in this paper is different from that of impending instability discussed in [6], [7]. The precursors in [6], [7] are intended to evaluate small-signal stability before its loss due to the change of system parameters. On the other hand, if the precursor of this paper is detected in a power system, then the system is regarded as already unstable in terms of the traditional all-or-nothing (that is, stable v.s. unstable) concept. In this paper, we are interested of how to monitor the course of short-term coupled swings leading to the loss of transient stability. For example, the CSI phenomenon which we address in this paper happens in multiple regimes: after a single disturbance to a power system, a group of synchronous generators in the system exhibits bounded, incoherent swings and then begins to coherently lose in synchronism with the rest of the system. In order to analyze and control this multi-scale phenomenon, it is inevitable to monitor the course of coupled swings before the de-synchronization is observed. For this purpose, the traditional all-or-nothing concept of transient stability is not effective. This is why we define a precursor to the loss of transient stability as an emergent transmission path of energy to one KM that represents the phenomenon of interest. This precursor gives a new measure for transient stability that can capture the course of short-term coupled swings.

Organization.-Sec. II poses two questions towards the goal of this paper. Sec. III reviews the existing theory of Koopman operator for nonlinear dynamical systems, including the definition of KM and its computation. Sec. IV develops analysis tools for the precursor identification: a new treatment of model reduction based on the $\mathrm{KM}$ and the action transfer operator. In Sec. V we apply the KM and action transfer operator to analysis of CSI in the New England 39-bus test system, and we demonstrate that they enable the identification of precursor to CSI. Sec. VI concludes this paper with a brief summary and remarks. A method for instability monitoring based on the results is described there.

Notation.-All vectors are viewed as columns. For a matrix A, we denote by $[\mathrm{A}]_{i j}$ the matrix entry in the $i$-th row and $j$-th column. The symbol $|z|$ stands for the magnitude of complexvalued variable $z, z^{\mathrm{c}}$ the complex conjugate of $z,\|\boldsymbol{x}\|$ the norm of vector $\boldsymbol{x}, \boldsymbol{x}^{\top}$ the transpose of $\boldsymbol{x}$, and $\boldsymbol{x}^{\dagger}$ the complexconjugate transpose of $\boldsymbol{x}$. We use $\mathbb{R}_{>0}:=\{x: x \in \mathbb{R}, x>0\}$ and $\mathbb{Z}_{\geq 0}:=\{0\} \cup \mathbb{N}$.

\section{Two Questions TOWARDS THE GOAL}

To set the goal of this paper, we pose two questions. Before this, we reconcile the notion of oscillatory instabilities used in power system studies [20], [21] with the context of classical mechanics. This paper deals with the loss of transient stability, and hence we do not consider instabilities of power system swing dynamics due to the change of system parameters which are discussed in [4]-[7]. Practical experience suggests that the dynamics of power system have a finite number of clean peaks in frequency spectrum, that is, it can be represented with a finite number of oscillatory modes. A relevant mathematical model is thus the dynamical system with a finite number $N$ of oscillatory modes, described by

$$
\left.\begin{array}{rr}
\frac{\mathrm{d} r_{i}}{\mathrm{~d} t}= & f_{i}\left(r_{1}, \ldots, r_{N}, \theta_{1}, \ldots, \theta_{N}\right), \\
\frac{\mathrm{d} \theta_{i}}{\mathrm{~d} t}=\Omega_{i}+g_{i}\left(r_{1}, \ldots, r_{N}, \theta_{1}, \ldots, \theta_{N}\right),
\end{array}\right\}
$$

where $i=1, \ldots, N$ denotes the integer label of modes, $r_{i}$ the amplitude of oscillatory mode $i, \theta_{i}$ its rotating phase, and $\Omega_{i}$ its angular frequency. The functions $f_{i}$ and $g_{i}$ define the interaction between different modes. The model (1) is one type of perturbations on $N$ degree of freedom integrable Hamiltonian systems [22]. With this model, we mathematically interpret the loss of transient stability. Since the dynamical system (1) is nonlinear, different initial conditions possibly induce different asymptotic behaviors. In particular, we may find that for one or some modes, the amplitudes grow in an unbounded way. This corresponds to the development of oscillatory instabilities (the growth of amplitudes of local, 
inter-machine, and inter-area mode oscillations; multi-swing instability; CSI, and so on) following a large but finite disturbance. The model (1) describes the connection between oscillatory instabilities and modal interactions, and hence it helps us to investigate precursors to the oscillatory instabilities. When attacking precursor issues using (1), we need to answer the two questions:

(Q1) How do we derive the model (1) for a practical power system model, in particular, when we consider dynamics that are far from a stable equilibrium in the model?

(Q2) How do we identify and monitor the emergence of an oscillatory instability using (1)?

Note that it is easy to obtain (1) for a linearized power system model using the standard modal matrix. In the rest of this paper, we will answer them for a nonlinear power system model, thereby suggesting a precursor to phenomena in loss of transient stability.

\section{NONLINEAR KOOPMAN MODES}

We provide an introduction to the theory of Koopman operator and Koopman Modes (KMs) for nonlinear dynamical systems. The contents here are based on [9]-[11]. The definitions of the Koopman operator and the KM are presented. The definition of coherency in the context of KM is also presented.

\section{A. Koopman Operator, Eigenvalue, and Mode}

In this section, we begin our argument by introducing the following continuous-time system evolving on a $n$ dimensional manifold $M$ : for $\boldsymbol{x} \in M$,

$$
\frac{\mathrm{d} \boldsymbol{x}}{\mathrm{d} t}=\boldsymbol{f}(\boldsymbol{x}),
$$

where $f$ is a nonlinear function defined on $M$. We assume that $\boldsymbol{f}$ belongs to $C^{1}(M)$, and its analytical expression is given. Here let us define a finite time- $h$ map $\boldsymbol{\Phi}_{t}^{t+h}$ for (2) as

$$
\boldsymbol{\Phi}_{t}^{t+h}: M \rightarrow M ; \boldsymbol{x}(t) \mapsto \boldsymbol{x}(t)+\int_{t}^{t+h} \boldsymbol{f}(\boldsymbol{x}(\tau)) \mathrm{d} \tau .
$$

Since (2) is autonomous, this map is time-invariant. Thus the discrete-time system on $M$ for constant time interval $h$ is defined as follows: for $\boldsymbol{x}[k]:=\boldsymbol{x}\left(t_{k}\right)$ and $h:=t_{k+1}-t_{k}$,

$$
\boldsymbol{x}[k+1]=\boldsymbol{\Phi}_{0}^{h}(\boldsymbol{x}[k]), \quad k \in \mathbb{Z}_{\geq 0} .
$$

We introduce the Koopman operator for the discrete-time system (4). The Koopman operator is a linear operator $\mathcal{U}$ that acts on scalar-valued functions on $M$ in the following manner: for any scalar-valued function $g: M \rightarrow \mathbb{R}, \mathcal{U}$ maps $g$ into a new function $\mathcal{U} g$ given by

$$
\mathcal{U} g(\boldsymbol{x})=g \circ \boldsymbol{\Phi}_{0}^{h}(\boldsymbol{x}) .
$$

Although the discrete-time system (4) can be given as a nonlinear map and evolve on a finite-dimensional manifold $M$, the Koopman operator $\mathcal{U}$ is linear, but infinite-dimensional. The eigenfunctions and eigenvalues of $\mathcal{U}$ are defined in the following manner: for scalar-valued functions $\varphi_{i}: M \rightarrow \mathbb{C}$ and constants $\lambda_{i} \in \mathbb{C}$,

$$
\mathcal{U} \varphi_{i}(\boldsymbol{x})=\lambda_{i} \varphi_{i}(\boldsymbol{x}), \quad i=1,2, \ldots
$$

We will refer to $\varphi_{i}$ as Koopman eigenfunctions and to $\lambda_{i}$ as the associated Koopman Eigenvalues (KEs).

The idea in [9] is to analyze nonlinear dynamics represented by (4), using the (linear) Koopman operator $\mathcal{U}$. By definition, the linear operator $\mathcal{U}$ does not rely on linearization and captures the full information of the nonlinear dynamics. To this end, consider a vector-valued observable $\boldsymbol{g}: M \rightarrow \mathbb{R}^{p}$. The function $\boldsymbol{g}(\boldsymbol{x})$ corresponds to a vector of any quantities of interest, such as frequencies and voltages metered at various points in a power system. In [9] the author shows that if the dynamical system (4) possesses a smooth invariant measure, or the initial condition $\boldsymbol{x}_{0}$ of (4) is on any (ergodic) attractor, then $\boldsymbol{g}(\boldsymbol{x}[k])=\left(g_{1}(\boldsymbol{x}[k]), \ldots, g_{p}(\boldsymbol{x}[k])\right)^{\boldsymbol{\top}}$ is exactly represented as follows:

$$
\begin{aligned}
\boldsymbol{g}(\boldsymbol{x}[k])= & \sum_{j=1}^{\infty} \lambda_{j}^{k} \varphi_{j}(\boldsymbol{x}[0]) \boldsymbol{v}_{j} \\
+ & {\left[\begin{array}{c}
\int_{0}^{2 \pi} \mathrm{e}^{\mathrm{i} k \theta} \mathrm{d} E(\theta) g_{1}(\boldsymbol{x}[0]) \\
\vdots \\
\int_{0}^{2 \pi} \mathrm{e}^{\mathrm{i} k \theta} \mathrm{d} E(\theta) g_{p}(\boldsymbol{x}[0])
\end{array}\right], }
\end{aligned}
$$

where $E(\theta)$ is a continuous, complex spectral measure. The modulus of KEs $\lambda_{i}$ is identically one, because $\mathcal{U}$ is a unitary operator in the above situation. In (5) we refer to the vectors $\boldsymbol{v}_{j}$ as Koopman Modes (KMs) of the system (4), corresponding to $\boldsymbol{g}$. On the right-hand side of (5), the first term represents the contribution of KEs (namely, discrete spectra of $\mathcal{U}$ ) to the time evolution $\{\boldsymbol{g}(\boldsymbol{x}[k])\}$ and describes the average and quasi-periodic parts of $\{\boldsymbol{g}(\boldsymbol{x}[k])\}$. On the other hand, the last term represents the contribution of continuous spectrum of $\mathcal{U}$ and describes the aperiodic part of $\{\boldsymbol{g}(\boldsymbol{x}[k])\}$. Hence, if the dynamics observed in (4) have no continuous spectrum in frequency domain (practical experience suggests this situation in power system analysis), then the dynamics are exactly represented as

$$
\boldsymbol{g}(\boldsymbol{x}[k])=\sum_{j=1}^{\infty} \lambda_{j}^{k} \varphi_{j}(\boldsymbol{x}[0]) \boldsymbol{v}_{j}
$$

In [9], [15], the authors show that the terms $\varphi_{j}(\boldsymbol{x}[0]) \boldsymbol{v}_{j}$ are defined and computed with a projection operation associated with $\mathcal{U}$ applied to the observable $\boldsymbol{g}$. Define a family of operators $\mathcal{P}^{\nu}$ : for $g: M \rightarrow \mathbb{R}$,

$$
\mathcal{P}^{\nu} g(\boldsymbol{x}[0])=\lim _{n \rightarrow \infty} \frac{1}{n} \sum_{k=0}^{n-1} \mathrm{e}^{-\mathrm{i} 2 \pi k \nu} g(\boldsymbol{x}[k]),
$$

where $\nu \in[-1 / 2,1 / 2)$. When the initial condition $\boldsymbol{x}[0]$ is on an (ergodic) attractor of (4), a nonzero $\mathcal{P}^{\nu}$ is the orthogonal projection operator onto the eigenspace of $\mathcal{U}$ associated with the $\mathrm{KE} \lambda=\mathrm{e}^{\mathrm{i} 2 \pi \nu}$. The projections of the $p$ components 
$g_{1}, \ldots, g_{p}$ of $\boldsymbol{g}$ on the $j$-th eigenspace are obtained:

$$
\left[\begin{array}{c}
\mathcal{P}^{\nu_{j}} g_{1}(\boldsymbol{x}[0]) \\
\vdots \\
\mathcal{P}^{\nu_{j}} g_{p}(\boldsymbol{x}[0])
\end{array}\right]=\varphi_{j}(\boldsymbol{x}[0]) \boldsymbol{v}_{j},
$$

where $\nu_{j}=\operatorname{Im}\left[\ln \lambda_{j}\right] / 2 \pi$. This formula (7) associates $\varphi_{j}(\boldsymbol{x}[0]) \boldsymbol{v}_{j}$ with the projection operation based on the nonzero operator $\mathcal{P}^{\nu}$. The left-hand sides of (7) are generalized Fourier transforms of observations $\{\boldsymbol{g}(\boldsymbol{x}[0]), \boldsymbol{g}(\boldsymbol{x}[1]), \ldots\}$, and hence the terms $\varphi_{j}(\boldsymbol{x}[0]) \boldsymbol{v}_{j}$ are directly computed. This implies that projections of any time series of nonlinear dynamics are obtained by generalized Fourier transforms at frequencies corresponding to the discrete spectra of $\mathcal{U}$. In this way, what we do here is not a simple FFT.

In the last paragraph, we have assumed that the dynamics of (4) are on an attractor. Even if this is not the case, that is, we consider dynamics off attractors of (4), the KM modes oscillate with a single frequency. If each of the $p$ components of $g$ lies within the span of eigenfunctions $\varphi_{j}$, then, as in [10], we may expand the vector-valued $g$ in terms of these eigenfunctions as

$$
\boldsymbol{g}(\boldsymbol{x})=\sum_{j=1}^{\infty} \varphi_{j}(\boldsymbol{x}) \boldsymbol{v}_{j},
$$

where $\boldsymbol{v}_{j}$ are also regarded as the coefficient vector in the expansion. The time evolution $\{\boldsymbol{g}(\boldsymbol{x}[k])\}$ starting at $\boldsymbol{g}(\boldsymbol{x}[0])$ is identically given by (6):

$$
\begin{aligned}
\boldsymbol{g}(\boldsymbol{x}[k]) & =\sum_{j=1}^{\infty} \varphi_{j}(\boldsymbol{x}[k]) \boldsymbol{v}_{j}=\sum_{j=1}^{\infty} \mathcal{U}^{k} \varphi_{j}(\boldsymbol{x}[0]) \boldsymbol{v}_{j} \\
& =\sum_{j=1}^{\infty} \lambda_{j}^{k} \varphi_{j}(\boldsymbol{x}[0]) \boldsymbol{v}_{j} .
\end{aligned}
$$

Thus we can refer to $\boldsymbol{v}_{j}$ as the KM which oscillates with a single frequency. If the dynamics observed here have only a finite number of discrete spectra in frequency domain, then we can expect that the expansion gives a good approximation of the dynamics. For dynamics off attractors, the KE $\lambda_{j}$ characterizes the temporal behavior of the corresponding KM $\boldsymbol{v}_{j}$ : the phase of $\lambda_{j}$ determines its frequency, and the magnitude determines the growth rate.

\section{B. Coherency in terms of Koopman Modes}

We define the notion of coherency in the context of KM. The case of oscillatory KM, in which the KE has a non-zero imaginary part, is addressed, because the study on coherency identification in power systems normally deals with oscillatory responses following a disturbance. For an oscillatory KM $\boldsymbol{v}_{i}$, called Mode $i$, with the $\operatorname{KE} \lambda_{i}=\left|\lambda_{i}\right| \mathrm{e}^{\mathrm{i} 2 \pi \nu_{i}}$ and its complex conjugate $\lambda_{i}^{\mathrm{c}}=\left|\lambda_{i}\right| \mathrm{e}^{-\mathrm{i} 2 \pi \nu_{i}}$, the associated modal dynamics $\boldsymbol{g}^{i}(\boldsymbol{x}[k])$ are given by

$$
\begin{aligned}
\boldsymbol{g}^{i}(\boldsymbol{x}[k]) & =\lambda_{i}^{k} \varphi_{i}(\boldsymbol{x}[0]) \boldsymbol{v}_{i}+\left(\lambda_{i}^{\mathrm{c}}\right)^{k}\left\{\varphi_{i}(\boldsymbol{x}[0]) \boldsymbol{v}_{i}\right\}^{\mathrm{c}} \\
& =2\left|\lambda_{i}\right|^{k}\left[\begin{array}{c}
A_{i 1} \cos \left(2 \pi k \nu_{i}+\alpha_{i 1}\right) \\
\vdots \\
A_{i p} \cos \left(2 \pi k \nu_{i}+\alpha_{i p}\right)
\end{array}\right],
\end{aligned}
$$

where

$$
A_{i j}:=\left|\left[\varphi_{i}(\boldsymbol{x}[0]) \boldsymbol{v}_{i}\right]_{j}\right|, \quad \alpha_{i j}:=\operatorname{Arg}\left(\left[\varphi_{i}(\boldsymbol{x}[0]) \boldsymbol{v}_{i}\right]_{j}\right) .
$$

Thus we can say that a set of oscillatory components $\mathbb{I} \subseteq$ $\{1, \ldots, m\}$ is coherent with respect to Mode $i$ if the amplitude coefficients $A_{i j}$ are the same for all $j \in \mathbb{I}$, and the initial phases $\alpha_{i j}$ are also the same. Note that the definition is strict compared with the definitions of slow-coherency [23], [24] and near-coherency [25], because it does not admit any finite, constant phase difference of oscillations along system evolution.

\section{AnALYSis ToOLS}

We develop analysis tools for the identification of precursors to instabilities of power systems. Tools include model reduction based on the Koopman Mode (KM) and PetrovGalerkin projective approximation (Sec. IV-A), action-angle representation of the reduced-order model (Sec.IV-B), and the action transfer operator quantifying transmission paths of energy between different KMs (Sec. IV-C). The way of deriving the action-angle representation gives the answer for (Q1).

\section{A. Petrov-Galerkin Projective Approximation}

We approximate the original dynamical system (4) by projecting the dynamics onto a low-dimensional subspace spanned by a small number of KMs. This will provide a reduced-order model for (4). Assume that the inverse of the observable $\boldsymbol{g}$ exists, denoted as $\boldsymbol{g}^{-1}$. This assumption is a mathematical generalization of observables available in power systems: indeed, it is sufficient that all of the states of a mathematical model are available (directly measured), namely, $\boldsymbol{g}$ is the identity. We use $\boldsymbol{V}_{i}:=\varphi_{i}(\boldsymbol{x}[0]) \boldsymbol{v}_{i}$ and view $\lambda_{i}^{k}$ as a time-dependent, complex-valued variable $z_{i}[k]$ below. The truncation of (8) after the first $m(<\infty)$ KMs gives

$$
\begin{aligned}
\boldsymbol{g}(\boldsymbol{x}[k]) & =\sum_{i=1}^{m} z_{i}[k] \boldsymbol{V}_{i}+\sum_{i=m+1}^{\infty} z_{i}[k] \boldsymbol{V}_{i} \\
& \approx \sum_{i=1}^{m} z_{i}[k] \boldsymbol{V}_{i} \\
& =\mathbf{V}_{m} \boldsymbol{z}^{m}[k],
\end{aligned}
$$

where $\vee_{m}:=\left(\boldsymbol{V}_{1}, \ldots, \boldsymbol{V}_{m}\right) \in \mathbb{C}^{p \times m}$ and $\boldsymbol{z}^{m}:=$ $\left(z_{1}, \ldots, z_{m}\right)^{\top} \in \mathbb{C}^{m}$. By substituting (11) into (4), we rewrite (4) as

$$
\sum_{i=1}^{m} z_{i}[k+1] \boldsymbol{V}_{i}=\left(\boldsymbol{g} \circ \boldsymbol{\Phi}_{0}^{h} \circ \boldsymbol{g}^{-1}\right)\left(\mathrm{V}_{m} \boldsymbol{z}^{m}[k]\right),
$$

and by multiplying each side of (12) by $\boldsymbol{V}_{j}^{\dagger}$ we have

$$
\begin{aligned}
\sum_{i=1}^{m} z_{i}[k+1] \boldsymbol{V}_{j}^{\dagger} \boldsymbol{V}_{i} & =\boldsymbol{V}_{j}^{\dagger}\left(\boldsymbol{g} \circ \boldsymbol{\Phi}_{0}^{h} \circ \boldsymbol{g}^{-1}\right)\left(\mathrm{V}_{m} \boldsymbol{z}^{m}[k]\right), \\
& =: \quad T_{j}\left(\boldsymbol{z}^{m}[k]\right),
\end{aligned}
$$

where $j=1, \ldots, m$, or

$$
\mathrm{E}_{m} \boldsymbol{z}^{m}[k+1]=\boldsymbol{T}_{m}\left(\boldsymbol{z}^{m}[k]\right),
$$


where $\mathrm{E}_{m}:=\mathrm{V}_{m}^{\dagger} \mathrm{V}_{m} \in \mathbb{C}^{m \times m}$ and $\boldsymbol{T}_{m}\left(\boldsymbol{z}^{m}\right):=$ $\left(T_{1}\left(\boldsymbol{z}^{m}\right), \ldots, T_{m}\left(\boldsymbol{z}^{m}\right)\right)^{\top} \in \mathbb{C}^{m}$. This discrete-time system (14) defined on $\mathbb{C}^{m}$ gives a reduced-order system of (4) in terms of the first $m$ KMs. This reduction can be done by a combination of practical data or simulation outputs to compute KMs and of a mathematical model.

\section{B. Action-Angle Representation}

When KMs are obtained from any set of observations, the KMs are completely isolated by definition and provide singlefrequency units of motion. This dynamically relevant property of KM is consistent with integrable Hamiltonian systems. Now we introduce the action-angle variables for the reduced-order system (14). For a regular $\mathrm{E}_{m}$, by defining $z_{j}=r_{j} \mathrm{e} \mathrm{e}^{\mathrm{i} \theta_{j}}$ where $\left(r_{j}, \theta_{j}\right) \in \mathbb{R}_{>0} \times \mathbb{T}$ for $j=1, \ldots, m$, we re-write (14) as

$$
r_{j}[k+1] \mathrm{e}^{\mathrm{i} \theta_{j}[k+1]}=\tilde{T}_{j}(\boldsymbol{r}[k], \boldsymbol{\theta}[k]),
$$

where $\boldsymbol{r}:=\left(r_{1}, \ldots, r_{m}\right)^{\top} \in \mathbb{R}_{>0}^{m}, \boldsymbol{\theta}:=\left(\theta_{1}, \ldots, \theta_{m}\right)^{\top} \in \mathbb{T}^{m}$, and

$$
\tilde{T}_{j}(\boldsymbol{r}, \boldsymbol{\theta}):=\sum_{i=1}^{m}\left[\mathrm{E}_{m}^{-1}\right]_{j i} T_{i}\left(r_{1} \mathrm{e}^{\mathrm{i} \theta_{1}}, \ldots, r_{m} \mathrm{e}^{\mathrm{i} \theta_{m}}\right) .
$$

The variable $r_{j}$ corresponds to the action variable in the Hamiltonian mechanics, and $\theta_{j}$ the angle variable. In fact, we can re-write (14) as

$$
\begin{aligned}
r_{j}[k+1] & =\sqrt{\tilde{T}_{j}^{\mathrm{c}}(\boldsymbol{r}[k], \boldsymbol{\theta}[k]) \tilde{T}_{j}(\boldsymbol{r}[k], \boldsymbol{\theta}[k])} \\
& =: F_{j}(\boldsymbol{r}[k], \boldsymbol{\theta}[k]), \\
\theta_{j}[k+1] & =\operatorname{Arg} \tilde{T}_{j}(\boldsymbol{r}[k], \boldsymbol{\theta}[k]) \\
& =: G_{j}(\boldsymbol{r}[k], \boldsymbol{\theta}[k]),
\end{aligned}
$$

or simply,

$$
\left.\begin{array}{l}
\boldsymbol{r}[k+1]=\boldsymbol{F}(\boldsymbol{r}[k], \boldsymbol{\theta}[k]), \\
\boldsymbol{\theta}[k+1]=\boldsymbol{G}(\boldsymbol{r}[k], \boldsymbol{\theta}[k]),
\end{array}\right\}
$$

where $\boldsymbol{F}(\boldsymbol{r}, \boldsymbol{\theta}):=\left(F_{1}(\boldsymbol{r}, \boldsymbol{\theta}), \ldots, F_{m}(\boldsymbol{r}, \boldsymbol{\theta})\right)^{\top}$ and $\boldsymbol{G}(\boldsymbol{r}, \boldsymbol{\theta}):=$ $\left(G_{1}(\boldsymbol{r}, \boldsymbol{\theta}), \ldots, G_{m}(\boldsymbol{r}, \boldsymbol{\theta})\right)^{\top}$. For the data on dynamics of (4) used for computation of the KMs, the action variables $r_{j}$ does not change as time goes on, and the angle variables $\theta_{j}$ rotate with constant speeds $\nu_{i}$ given by the KEs. In this way, we can regard the right-hand sides of (15) as terms in an actionangle formulation of perturbed Hamiltonian systems [22]. The model (15) is the analog of (1) for the nonlinear discrete-time system (4). Thus we have the answer for (Q1).

\section{Action Transfer Operator}

In [19] the authors gave an insight into the transmission paths of energy in a nearly-integrable Hamiltonian system by linearizing the action-angle representation of the system. The linearized system provides the time-dependent operator as the system evolves. In this paper we term this operator the action transfer operator that can quantify the change of action variables and will be defined below. Now we apply the approach developed in [19] to the discrete-time system (15) and show that by means of $\mathrm{KM}$, the action transfer operator is

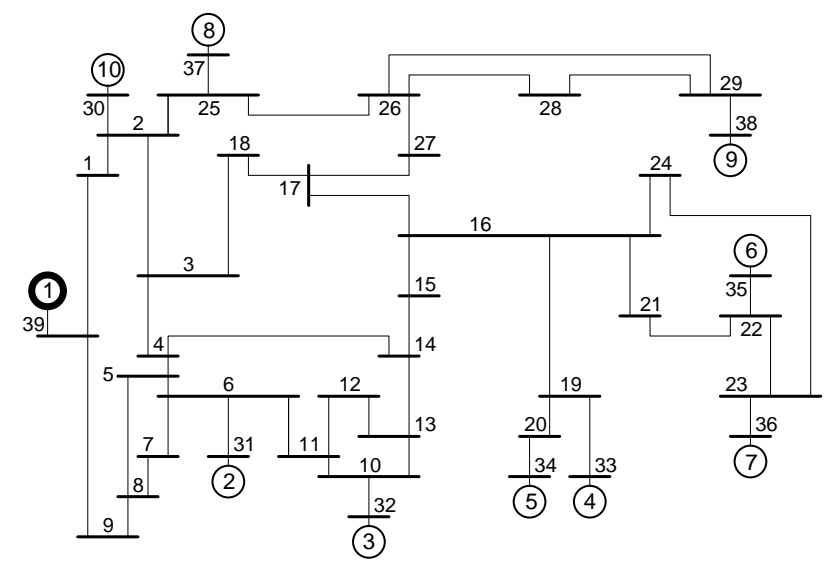

Fig. 1. The New England 39-bus test system [26], [27]

defined for arbitrary dynamical systems. The linearizd system of (15) around a point $\left(\boldsymbol{r}^{*}, \boldsymbol{\theta}^{*}\right)$ is given by

$$
\left[\begin{array}{c}
\boldsymbol{r}[k+1] \\
\boldsymbol{\theta}[k+1]
\end{array}\right]=\left.\left[\begin{array}{cc}
\mathrm{D}_{r} \boldsymbol{F} & \mathrm{D}_{\theta} \boldsymbol{F} \\
\mathrm{D}_{r} \boldsymbol{G} & \mathrm{D}_{\theta} \boldsymbol{G}
\end{array}\right]\right|_{\left(\boldsymbol{r}^{*}, \boldsymbol{\theta}^{*}\right)}\left[\begin{array}{c}
\boldsymbol{r}[k] \\
\boldsymbol{\theta}[k]
\end{array}\right] .
$$

Since the action variables are often associated with amount of energy contained in every KMs, the sub-matrix $\mathrm{D}_{r} \boldsymbol{F}\left(\boldsymbol{r}^{*}, \boldsymbol{\theta}^{*}\right)$ provides a quantitative index of the model interaction via energy. For simplicity we assume $\boldsymbol{g}(\boldsymbol{x})=\boldsymbol{x}$. Then we can define the Jacobi matrix $\mathrm{J}(\boldsymbol{x}[k])$ that describes the infinitesimal change of $\boldsymbol{r}[k]$ at a point $\boldsymbol{x}[k]$ :

$$
\mathrm{J}(\boldsymbol{x}[k]):=\mathrm{D}_{r} \boldsymbol{F}(\boldsymbol{x}[k])-\mathrm{I}_{m}
$$

where $\mathrm{I}_{m}$ is the identity matrix of size $m$. The right-hand side of (16) can be computed numerically: see Appendix for details. The Jacobi matrix is a time-dependent operator and quantifies the change of action variables for the $m$ KMs. We term the Jacobi matrix the action transfer operator for the KMs. In the next section, by using a concrete example of power system analysis, we will illustrate that the action transfer operator works for the identification of precursor to loss of transient stability.

\section{A Precursor to Loss of Transient Stability}

We apply the Koopman Mode (KM) Analysis and tools developed in Sec. IV to analysis of coupled swings in the New England 39-bus test system (NE system) [26], [27]. The NE system is shown in Fig. 1 and consists of 10 synchronous generators, 39 buses, loads, and AC transmission lines. Here we focus on one important type of loss of transient stability, the Coherent Swing Instability (CSI) (see Sec. I for definition and Sec. V-A for phenomenology). We show that the CSI phenomenon follows an emergent transmission path of energy from high-frequency KMs to the lowest KM with flat basis vector, and that the emergent transmission path can be detected by computation of the action transfer operator. This is the answer for (Q2) and corresponds to the main result of this paper. 


\section{A. Coherent Swing Instability}

We introduce the equations of motion for the NE system. Assume that bus 39 is the infinite bus. The short-term swing dynamics of generators $2-10$ are represented by the swing equations [20], [27]:

$$
\begin{aligned}
\frac{H_{i}}{\pi f_{\mathrm{s}}} \frac{\mathrm{d}^{2} \delta_{i}}{\mathrm{~d} t^{2}}+D_{i} \frac{\mathrm{d} \delta_{i}}{\mathrm{~d} t}= & P_{\mathrm{m} i}-G_{i i} E_{i}^{2}-\sum_{j=1, j \neq i}^{10} E_{i} E_{j} . \\
& \cdot\left\{G_{i j} \cos \left(\delta_{i}-\delta_{j}\right)\right. \\
& \left.+B_{i j} \sin \left(\delta_{i}-\delta_{j}\right)\right\},
\end{aligned}
$$

where the integer label $i=2, \ldots, 10$ denotes generator $i$. The variable $\delta_{i}$ is the angular position of rotor in generator $i$ with respect to bus 1 and is in radian. We set the variable $\delta_{1}$ to a constant because bus 1 is assumed to be the infinite bus. The parameters $f_{\mathrm{s}}, H_{i}, D_{i}, P_{\mathrm{m} i}, E_{i}, G_{i i}, G_{i j}$, and $B_{i j}$ are in per unit system except for $H_{i}$ and $D_{i}$ in second, and for $f_{\mathrm{s}}$ in Hertz. The mechanical input power $P_{\mathrm{m} i}$ to generator $i$ and the internal voltage $E_{i}$ of generator $i$ are normally constant for transient stability analysis [20]. The parameter $H_{i}$ is the per unit time inertia constant of generator $i$, and $D_{i}$ its damping coefficient. The parameter $G_{i i}$ is the internal conductance, and $G_{i j}+\mathrm{i} B_{i j}$ is the transfer admittance between generators $i$ and $j$. Electrical loads are modeled as lumped admittances in order to derive (17).

The setting of numerical simulation is as follows. The voltage $E_{i}$ and a stable equilibrium $\left(\delta_{i}^{*}, \omega_{i}^{*}=0\right)$ for generator $i$ are fixed using power flow computation [20]. The constants $H_{i}, P_{\mathrm{m} i}$, and power loads are the same as in [27]. The parameter $D_{i}$ is fixed at $0 \mathrm{~s}$, and $f_{\mathrm{b}}$ at $60 \mathrm{~Hz}$. We will consider a case of non-zero dissipation in Sec. V-D. The elements $G_{i i}$, $G_{i j}$, and $B_{i j}$ are calculated using the data in [27] and the power flow computation. All numerical simulations discussed in this section were performed using MATLAB: the function ode 45 is used for integration of (17).

We present an example of coupled swings in the NE system. Fig. 2 shows the time responses of angular positions $\delta_{i}$ of 9 generators in the NE system under the two initial conditions: for $\omega_{i}=\mathrm{d} \delta_{i} / \mathrm{d} t$,

$$
\left(\delta_{i}(0), \omega_{i}(0)\right)= \begin{cases}\left(\delta_{i}^{*}+1.000 \mathrm{rad}, 3 \mathrm{rad} / \mathrm{s}\right) & i=8, \\ \left(\delta_{i}^{*}, 0 \mathrm{rad} / \mathrm{s}\right) & \text { else },\end{cases}
$$

and

$$
\left(\delta_{i}(0), \omega_{i}(0)\right)= \begin{cases}\left(\delta_{i}^{*}+1.575 \mathrm{rad}, 3 \mathrm{rad} / \mathrm{s}\right) & i=8, \\ \left(\delta_{i}^{*}, 0 \mathrm{rad} / \mathrm{s}\right) & \text { else. }\end{cases}
$$

The initial conditions physically correspond to local disturbances at generator 8 . Note that the initial perturbations are artificial, but we can find by the use of short circuit the same phenomenon as in Fig. 2(b) (see [18]). In Fig. 2(a) the generators do not show any stepping-out, that is, they do not show any loss of transient stability for the selected disturbance. Generators 2, 3, 6, and 7 show a coherent swing excited by the local disturbance. We call these generators the coherent group (see [11]). The other generators show incoherent swings in Fig. 2(a). Generator 9 shows a swing similar in frequency and phase to the coherent group, but the swing amplitude is a little (a)
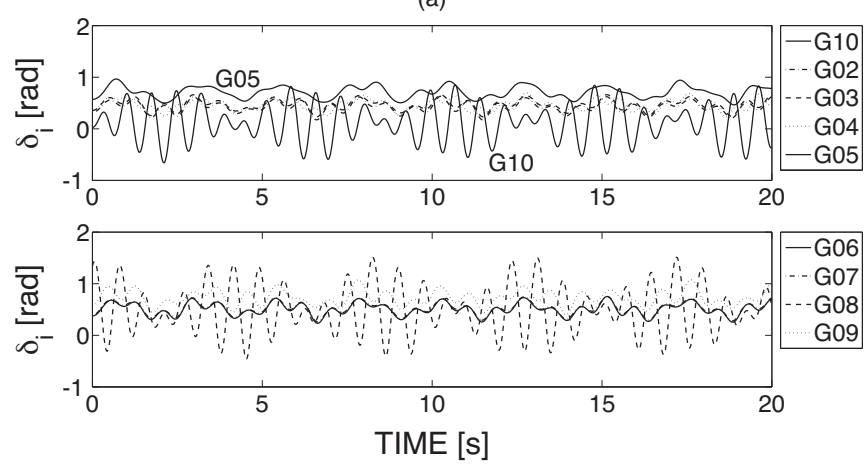

(b)
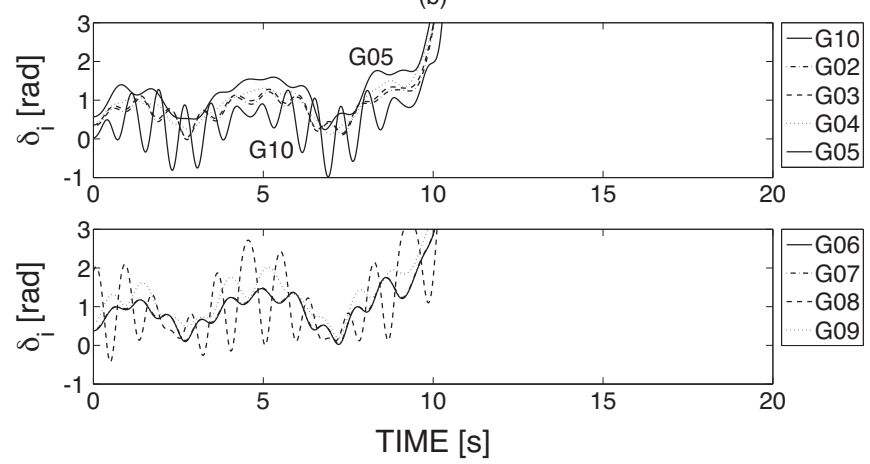

Fig. 2. Coupled swings and instability observed in in the New England 39-bus test system $\left(D_{i}=0\right)$ : (a) the initial condition (18) and (b) the initial condition (19). The angular positions $\delta_{i}$ only are displayed.

larger. Generators 8 and 10 have swings of larger amplitudes than the others, because the initial conditions are localized at generator 8 , and the two generators are electrically close. On the other hand, in Fig. 2(b) they are bounded during the period from $t=0 \mathrm{~s}$ to $7 \mathrm{~s}$ and then begin to grow coherently. Every generator loses synchronism with the infinite bus at the same time. This corresponds to the growth of amplitude of interarea mode oscillation between the NE system and the infinite bus, namely, the outside of the system. This is typical of the CSI phenomenon.

In [18] we showed that CSI involves the divergent motion in the projection of full-system dynamics onto the plane of collective variables. The collective variables correspond to the well-known COA (Center-Of-Angle) coordinates (see [26], [27] for introduction). For the NE system, the $\mathrm{COA} \delta_{\mathrm{COA}}$ and its time derivative $\omega_{\mathrm{COA}}$ are defined as

$$
\delta_{\mathrm{COA}}:=\sum_{i=2}^{10} \frac{H_{i}}{H} \delta_{i}, \omega_{\mathrm{COA}}:=\frac{\mathrm{d} \delta_{\mathrm{COA}}}{\mathrm{d} t}=\sum_{i=2}^{10} \frac{H_{i}}{H} \omega_{i},
$$

where $H:=\sum_{i=2}^{10} H_{i}$. The variables $\delta_{\mathrm{COA}}$ and $\omega_{\mathrm{COA}}$ describe the averaged motion of all the generators in the system. Fig. 3 plots the trajectories of (17) shown in Fig. 2 on $\delta_{\mathrm{COA}}-\omega_{\mathrm{COA}}$ plane. The trajectories start near the origin at time $0 \mathrm{~s}$ and make a couple of almost periodic loops around the initial point. In Fig. 3(a) the trajectory keeps executing bounded loops. In Fig. 3(b) it escapes and finally diverges.

Here, based on [18], we briefly describe the dynamical mechanism of CSI. This phenomenon occurs in a high- 

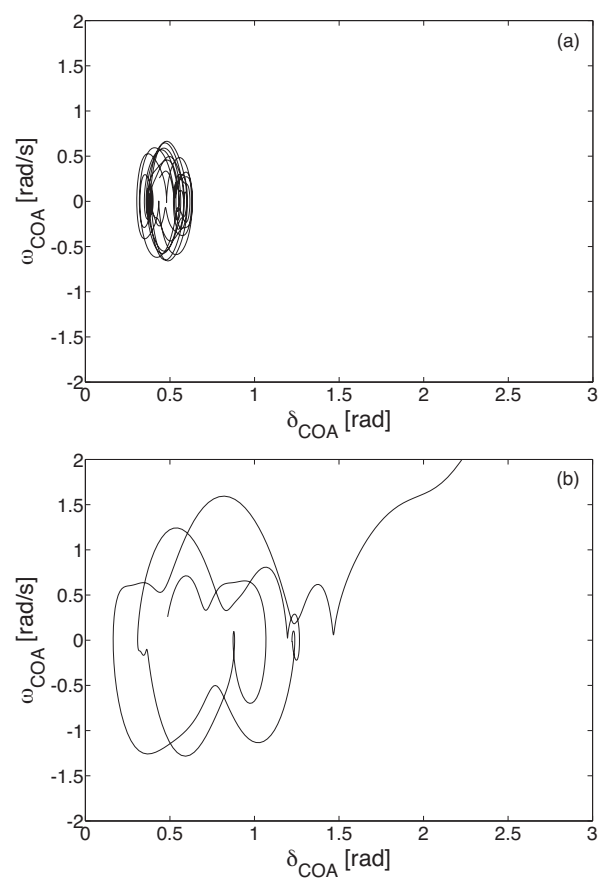

Fig. 3. Collective dynamics in the New England system $\left(D_{i}=0\right)$ : (a) the initial condition (18) and (b) the initial condition (19). The Center-Of-Angle (COA) coordinates are defined in (20).

dimensional oscillatory system with dominant inertia in which the full dynamics are decomposed into many strong linear oscillatory modes and one weak nonlinear mode. The modal decomposition is possible by the classical linear analysis for a nearly solvable loop power grid [18], and the proper orthonormal decomposition and the KM Analysis for the NE system [11]. Note that the dynamics of the weak nonlinear mode are captured by the COA coordinates. Then strong linear oscillations act as perturbations to the nonlinear collective mode. This is analytically justified with partial averaging in a similar oscillatory system [19]. Also the averaging technique suggests that the effect of linear oscillations to the nonlinear mode is from higher frequency to lower frequency modes. This is why a precursor identified in this paper is generally expected for this sort of instability.

\section{B. Computation of Koopman Modes}

We compute the KMs for the coupled swings shown in Fig. 2(a). The computation of KMs is done with the Fourierbased formula (7). For computation we need to choose the observable $\boldsymbol{g}(\boldsymbol{x})$ where $\boldsymbol{x}=\left(\delta_{2}, \ldots, \delta_{10}, \omega_{2}, \ldots, \omega_{10}\right)^{\mathrm{T}}$. In this paper we use the variable $\boldsymbol{x}$ as the observable, namely $\boldsymbol{g}(\boldsymbol{x})=\boldsymbol{x}$. We use the simulation output shown in Fig. 2(a) that extracts the data $\{\boldsymbol{x}(n T)\}_{n=0}^{N}$, where the uniform sampling period $T=1 /(50 \mathrm{~Hz})$ and the number of samples $N+1=1001$. Also, in order to use (7) for computing KMs, we need to identify dominant frequencies in the course of coupled swings. DFT (Discrete Fourier Transform) analysis shows that the time responses in Fig. 2(a) have clean peaks at the five frequencies, $0.40 \mathrm{~Hz}, 0.90 \mathrm{~Hz}, 1.00 \mathrm{~Hz}, 1.25 \mathrm{~Hz}$, and $1.45 \mathrm{~Hz}$.
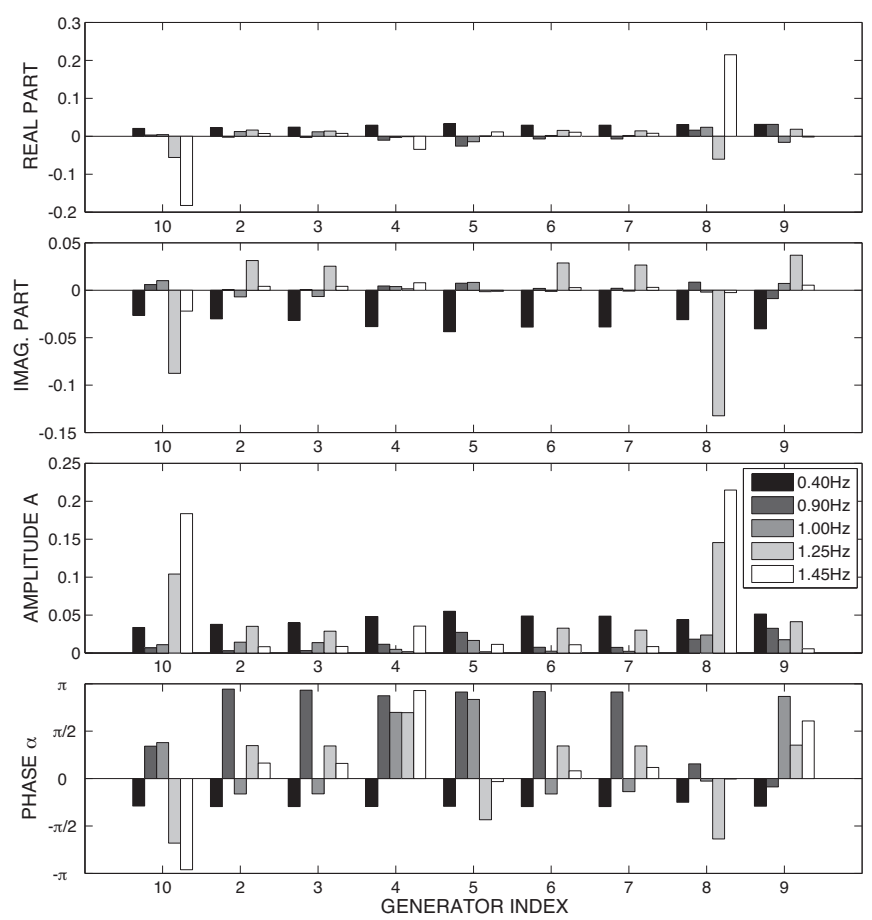

Fig. 4. Computation of Koopman modes $\left(\boldsymbol{V}_{i}=\varphi_{i}(\boldsymbol{x}[0]) \boldsymbol{v}_{i}\right)$ using the projection operator $\mathcal{P}^{\nu}$ under $\nu=(0.40 \mathrm{~Hz}) T$ at $i=1,(0.90 \mathrm{~Hz}) T$ at $i=2,(1.00 \mathrm{~Hz}) T$ at $i=3,(1.25 \mathrm{~Hz}) T$ at $i=4$, and $(1.45 \mathrm{~Hz}) T$ at $i=5$. Here the symbol $T$ is the sampling period. The amplitude coefficients $A_{j i}$ and initial phases $\alpha_{j i}$, defined in (9), are also shown. These are obtained for the waveform in Fig. 2(a).

Now we compute the terms $\varphi_{i}(\boldsymbol{x}[0]) \boldsymbol{v}_{i}$ using the projection operator $\mathcal{P}^{\nu}$ with the five frequencies: $i=1$ for $0.40 \mathrm{~Hz}, 2$ for $0.90 \mathrm{~Hz}, 3$ for $1.00 \mathrm{~Hz}, 4$ for $1.25 \mathrm{~Hz}$, and 5 for $1.45 \mathrm{~Hz}$. We use the finite-time approximation of (7) from $k=0$ to $N$, where $N+1$ is the number of samples. The numerical results are shown in Fig. 4. The angular positions $\delta_{i}$ only are displayed. The amplitude coefficients and initial phases, which are defined in (9), are also shown. For $0.40 \mathrm{~Hz}$, the values of amplitude coefficients are close for each of the generators, and their initial phases are also close. All the swings of the 9 generators are hence said to be coherent with respect to the $\mathrm{KM}$ with $0.40 \mathrm{~Hz}$. We call it the coherent KM. For $1.25 \mathrm{~Hz}$ and $1.45 \mathrm{~Hz}$, the amplitude coefficients for generators 8 and 10 are larger than the others. These two KMs capture the large swings of generators 8 and 10 shown in Fig. 2(a). Thus we can extract spatial modes oscillating with a single frequency.

\section{A Precursor to Coherent Swing Instability}

We compute the action transfer operator $\mathrm{J}$ for the CSI phenomenon shown in Fig. 2(b). The KMs in Fig. 4 are computed for the bounded swings in Fig. 2(a). The results on Figs. 2(a) and (b) are obtained with different initial conditions of (17). Hence we consider how J behaves under dynamics perturbed by a slight change of initial conditions. We use the tools (model decomposition and action-angle representation) developed in Sec. IV and the formulas presented in Appendix for computation. 


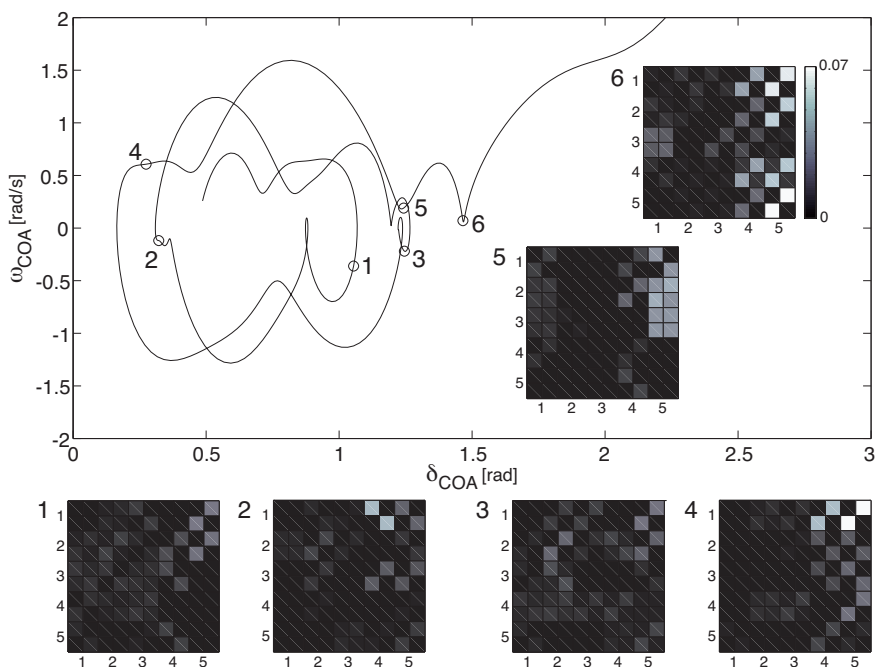

Fig. 5. Collective dynamics of the CSI phenomenon shown in Fig. 3(b) and snapshots of the Jacobi matrix J. The six snapshots are at time (1) $1.2 \mathrm{~s},(2)$ $2.8 \mathrm{~s}$, (3) $5.0 \mathrm{~s}$, (4) $7.2 \mathrm{~s}$, (5) $8.4 \mathrm{~s}$, and (6) $9.0 \mathrm{~s}$.

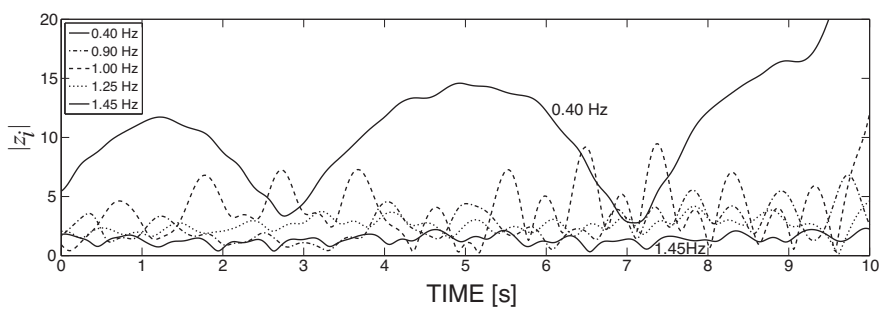

Fig. 6. Time evolution of magnitude $\left|z_{i}\right|$ for the five Koopman modes. This is associated with the time evolution of the action transfer operator $\mathrm{J}$ shown in Fig. 5.

Figure 5 shows several snapshots of the action transfer operator $\mathrm{J}$ along the COA trajectory shown in Fig. 3(b). In the snapshots the $i$-th $\mathrm{KM}(i=1, \ldots, 5)$ corresponds to the $2 i-1$ and $2 i$ rows. The two variables $z_{2 i-1}=r_{2 i-1} \mathrm{e}^{\mathrm{j} \theta_{2 i-1}}$ and $z_{2 i}=r_{2 i} \mathrm{e}^{\mathrm{j} \theta_{2 i}}$ are assigned to the $i$-th KM. By definition the action variables $r_{2 i-1}$ and $r_{2 i}$ have the same behaviors, and the angle variables $\theta_{2 i-1}$ and $-\theta_{2 i}$ also have the same. Because both the variables $r_{2 i-1}$ and $r_{2 i}$ affect the infinitesimal change of $r_{j}(j=1, \ldots, 10)$, in $\mathrm{J}$ we need to plot $\partial F_{j} / \partial r_{2 i-1}$ as well as $\partial F_{j} / \partial r_{2 i} \cdot{ }^{1}$ First, the magnitude of elements in the upper triangular part change as time goes on. On the other hand, the magnitude of elements in the lower triangular part does not change and is almost constant. This salient feature of $\mathrm{J}$ suggests directed transmission paths of energy from highfrequency KMs to low ones. This is consistent with that in [19] that analyzes global dynamics occurring in nearly-integrable Hamiltonian systems. The current result is obtained for a set of KMs embedded in dynamics of a general power grid model with nonlinear local interconnections. Second, we see that the transmission path of energy from high-frequency KMs to the

${ }^{1}$ See Fig. 5 . For the 1st KM $(j=1)$, in the snapshot 2 the variable $r_{9}$ for the 5th KM dominantly affects the time change of $r_{1}$. On the other hand, in the snapshot 4 the variable $r_{10}$ dominantly affects $r_{1}$. These observations both are regarded as the effect of the 5th KM to the 1st KM.

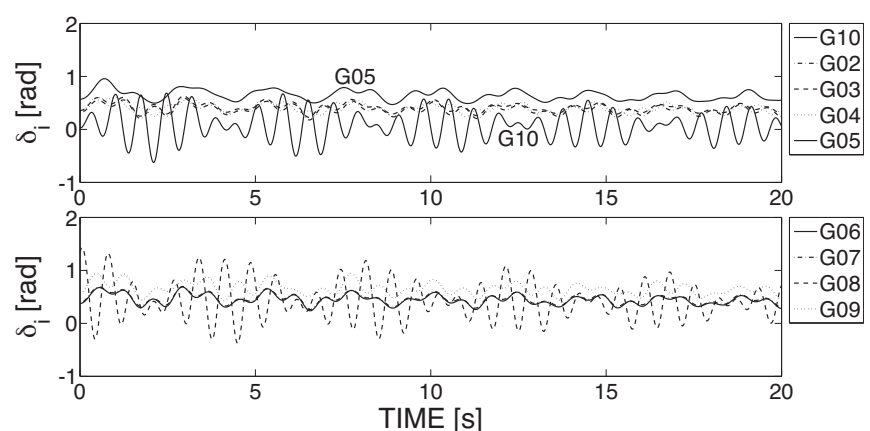

(a)

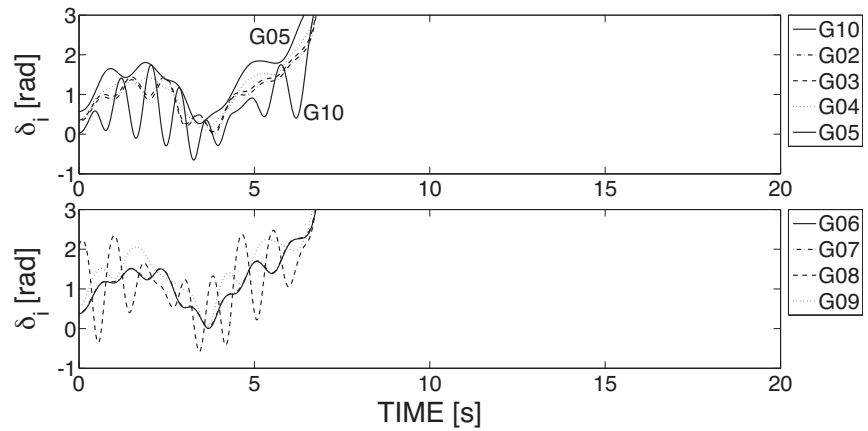

(b)

Fig. 7. Coupled swings and instability observed in in the New England 39bus test system $\left(D_{i}=0.01\right)$ : (a) the initial condition (21) and (b) the initial condition (22). The angular positions $\delta_{i}$ only are displayed.

lowest one becomes stronger when the COA trajectory largely grows in the neighborhood of the $\delta_{\mathrm{COA}}$-axis: see the snapshots at time (4) $7.2 \mathrm{~s}$ and (6) $9.0 \mathrm{~s}$ in Fig. 5. The transmission path appears in several times, and then the magnitude $\left|z_{1}\right|$ of the lowest KM begins to diverge as shown in Fig. 6 while the magnitudes of the other KMs are still bounded. Since the lowest $\mathrm{KM}$ with $0.40 \mathrm{~Hz}$ is the coherent $\mathrm{KM}$, the divergence of magnitude $\left|z_{1}\right|$ implies CSI. This provides a precursor diagnostic for CSI: the emergent transmission path of energy from high-frequency KMs to the lowest, coherent KM.

\section{On the Effect of Non-Zero Dissipation}

Finally we discuss the effect of non-zero dissipation on the time evolution of action transfer operator. Here we fix the damping parameter $D_{i}$ at 0.01 . Then two types of coupled dynamics in the 9 generators are observed: bounded swings and the CSI phenomenon. Fig. 7 shows the coupled swings and instability. We use the following two different sets of initial conditions:

$$
\left(\delta_{i}(0), \omega_{i}(0)\right)= \begin{cases}\left(\delta_{i}^{*}+1.00 \mathrm{rad}, 3 \mathrm{rad} / \mathrm{s}\right) & i=8, \\ \left(\delta_{i}^{*}, 0 \mathrm{rad} / \mathrm{s}\right) & \text { else },\end{cases}
$$

and

$$
\left(\delta_{i}(0), \omega_{i}(0)\right)= \begin{cases}\left(\delta_{i}^{*}+1.75 \mathrm{rad}, 3 \mathrm{rad} / \mathrm{s}\right) & i=8, \\ \left(\delta_{i}^{*}, 0 \mathrm{rad} / \mathrm{s}\right) & \text { else. }\end{cases}
$$

In Fig. 7(a) we can pick up four dominant frequencies: $0.40 \mathrm{~Hz}, 1.00 \mathrm{~Hz}, 1.25 \mathrm{~Hz}$, and $1.45 \mathrm{~Hz}$. By computing the KMs for these frequencies in the same way as Sec. V-B, we 


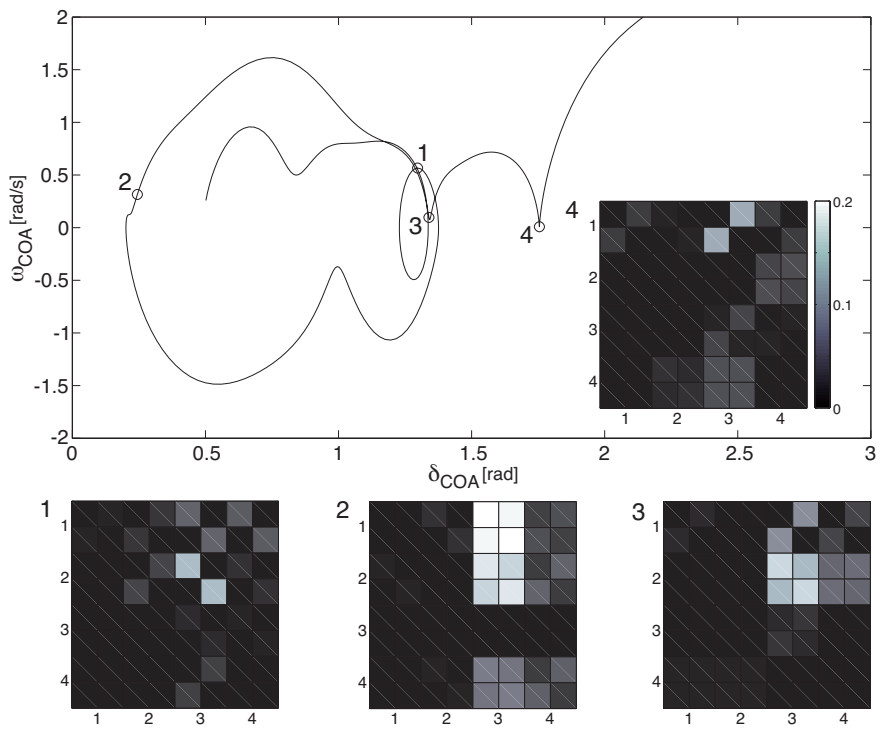

Fig. 8. Collective dynamics of the CSI phenomenon shown in Fig. 7(b) and snapshots of the Jacobi matrix J. Four KMs $(0.40 \mathrm{~Hz}, 1.00 \mathrm{~Hz}, 1.25 \mathrm{~Hz}$, and $1.45 \mathrm{~Hz}$, numbered with 1 to 4 ) are obtained with the result of numerical simulation in Fig. 7(a) and are used for the computation of J. The snapshots are at (1) $1.8 \mathrm{~s},(2) 3.8 \mathrm{~s},(3) 5.0 \mathrm{~s}$, and (4) $6.0 \mathrm{~s}$

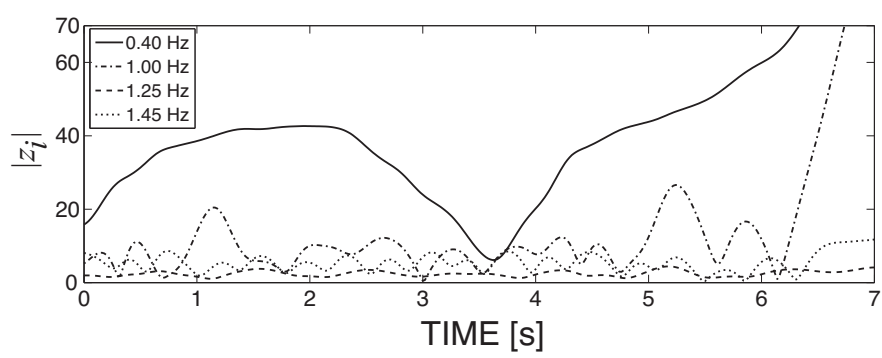

Fig. 9. Time evolution of magnitude $\left|z_{i}\right|$ for the four Koopman modes. This is associated with the time evolution of the action transfer operator $\mathrm{J}$ shown in Fig. 8.

compute the action transfer operator for the CSI phenomenon. Note that the lowest KM with $0.40 \mathrm{~Hz}$ has almost flat basis vector and corresponds to the coherent mode in this simulation. Fig. 8 shows several snapshots of $\mathrm{J}$ along the COA trajectory calculated for the result in Fig. 7(b). First, we see the transmission path of energy to the lowest KM. The transmission path appears in several times, and then the magnitude $\left|z_{1}\right|$ of the lowest KM diverges while the magnitudes of the other KMs are bounded: compare the snapshots at time (2) $3.8 \mathrm{~s}$ and (4) $6.0 \mathrm{~s}$ in Fig. 8 with Fig. 9. This is consistent with that in the case of zero dissipation. Thus we say that the transmission path of energy can survive as a precursor. Second, in contrast with Fig. 5, we see a few strong elements in the lower triangular part. This suggests that a transmission path of energy from low KMs to high ones can emerge. The origin of this is the existence of non-zero uniform dissipation. In the case of $D_{i}=0.01$, the transmission path of energy due to the system structure overwhelms that due to dissipation, and thus the CSI phenomenon emerges against the perturbation of small dissipation.

\section{SUMMARY AND REMARKS}

In this paper we identified a precursor to phenomena of loss of transient stability in multi-machine power systems via the so-called Coherent Swing Instability (CSI) that corresponds to the emergent transmission path of energy from high-frequency Koopman Modes (KMs) to the lowest frequency KM. The KM analysis enables the decomposition of nonlinear dynamic phenomena into dynamically relevant modes and the refinement of mathematical model of the CSI that is suitable for identifying the emergent energy transmission. Hence the KM Analysis plays an important role in identifying the precursor. Also we illustrated that the precursor persists under a perturbation consisting of small dissipation.

Several remarks related to these are provided. Firstly, using the action transfer operator, we quantified and visualized an interaction between nonlinear KMs that is due to system nonlinearity as well as slight changes of initial conditions. This is a mechanism different from that in [4], [5] which addresses an interaction between linear modes due to system nonlinearity. The use of linear modes is valid because they analyze long-term swing dynamics. On the other hand, since we analyze the loss of transient stability as a nonlocal phenomenon, the use of KMs is relevant in the current analysis. Secondly, in order to monitor the dynamics of power system using this precursor, we need to monitor evolution of state of the power system to enable the computation of KMs. Such data can be obtained from an off-line transient simulation as well as on-line metering. Lastly, the precursor of this paper is developed specifically for the case of CSI. Our analysis tools in Sec. IV are based on the general nonlinear dynamical system (2). Hence it is possible to include static or dynamic models of loads that may affect this type of instability. Other phenomena in loss of transient stability could be examined using the KM Analysis as well, and structural changes in the action transfer operator could be sought to devise instability precursors. Under non-small dissipation, a transmission path of energy from low-frequency KM to high-frequency KM may be quantified via the KM Analysis: one glimpse of this is shown in Fig. 8. Thus, the KM Analysis leads to a potentially broader set of applications for instability monitoring of power systems.

Before closing this paper, we describe a method for instability monitoring of power systems by a combination of practical data (possibly, numerical simulations), mathematical modeling, and computation. Enabling tools for the purpose lead to radical change in how to monitor dynamic phenomena of the power system. The monitoring process which we claim here consist of the three stages:

(i) Method for characterizing a dynamic phenomenon, where practical data measured in a real power system is decomposed into modes that capture the phenomenon of interest.

(ii) Method for refining a mathematical model of the power system, where it is represented in a new coordinate system that is better for instability monitoring.

(iii) Method for instability monitoring of the power system by a combination of practical data, the refined model, and 
computation.

Methods (i) and (ii) are possible by the KM Analysis and the model refinement developed in Secs. IV-A and IV-B. Method (iii) is based on the precursor whose identification is possible by computing the action transfer operator in Sec. IV-C along given time series. Validating the monitoring method is in future directions.

\section{ACKNOWLEDGEMENTS}

We appreciate Professor Petar V. Kokotović (The University of California, Santa Barbara) for his suggestive question on precursors to power system instabilities and Professor Takashi Hikihara (Kyoto University) for his constant support of this work. We also thank anonymous reviewers for their suggestions and comments.

\section{APPENDIX}

\section{Formulas FOR COMPUTATION OF THE ACTION TRANSFER OPERATOR}

Assume that the observable $\boldsymbol{g}$ is the identity map, i.e. $\boldsymbol{g}(\boldsymbol{x})=\boldsymbol{x}$. We derive the analytical formulas that enable the computation of $\mathrm{J}=\mathrm{D}_{r} \boldsymbol{F}-\mathrm{I}_{m}$ : first,

$$
\begin{aligned}
\frac{\partial F_{j}}{\partial r_{i}}(\boldsymbol{r}, \boldsymbol{\theta}) & =\frac{\partial}{\partial r_{i}} \sqrt{\tilde{T}_{j}^{\mathrm{c}} \tilde{T}_{j}} \\
& =\frac{1}{2 \sqrt{\tilde{T}_{j}^{\mathrm{c}} \tilde{T}_{j}}}\left(\tilde{T}_{j}^{\mathrm{c}} \frac{\partial \tilde{T}_{j}}{\partial r_{i}}+\tilde{T}_{j} \frac{\partial \tilde{T}_{j}^{\mathrm{c}}}{\partial r_{i}}\right) \\
& =\frac{1}{F_{j}(\boldsymbol{r}, \boldsymbol{\theta})} \operatorname{Re}\left[\tilde{T}_{j}^{\mathrm{c}}(\boldsymbol{r}, \boldsymbol{\theta}) \frac{\partial \tilde{T}_{j}}{\partial r_{i}}(\boldsymbol{r}, \boldsymbol{\theta})\right] .
\end{aligned}
$$

The derivative term of $\tilde{T}_{j}$ with respect to $r_{i}$ on the right-hand side of (23) is the following:

$$
\begin{aligned}
\frac{\partial \tilde{T}_{j}}{\partial r_{i}}(\boldsymbol{r}, \boldsymbol{\theta}) & =\frac{\partial z_{i}}{\partial r_{i}} \frac{\partial \tilde{T}}{\partial z_{i}}(\boldsymbol{z}) \\
& =\mathrm{e}^{\mathrm{i} \theta_{i}} \sum_{l=1}^{m}\left[\mathrm{E}_{m}^{-1}\right]_{j l} \frac{\partial T_{l}}{\partial z_{i}}(\boldsymbol{z}) \\
& =\mathrm{e}^{\mathrm{i} \theta_{i}} \sum_{l=1}^{m}\left[\mathrm{E}_{m}^{-1}\right]_{j l} \boldsymbol{V}_{l}^{\dagger} \frac{\partial \boldsymbol{\Phi}_{0}^{h}}{\partial z_{i}}(\boldsymbol{z}) \\
& =\mathrm{e}^{\mathrm{i} \theta_{i}} \sum_{l=1}^{m}\left[\mathrm{E}_{m}^{-1}\right]_{j l} \boldsymbol{V}_{l}^{\dagger} \sum_{q=1}^{n} V_{q i} \frac{\partial \boldsymbol{\Phi}_{0}^{h}}{\partial x_{q}}(\boldsymbol{x}) .
\end{aligned}
$$

The spatial derivative $\partial \boldsymbol{\Phi}_{0}^{h} / \partial x_{q}$ at point $\boldsymbol{x}[k]$ is approximated with, for example,

$$
\begin{aligned}
\frac{\partial \boldsymbol{\Phi}_{0}^{h}}{\partial x_{q}}(\boldsymbol{x}[k]) \approx & \frac{1}{2 \Delta x}\left\{\boldsymbol{\Phi}_{0}^{h}\left(\boldsymbol{x}[k]+\boldsymbol{e}_{q} \Delta x\right)\right. \\
& \left.-\boldsymbol{\Phi}_{0}^{h}\left(\boldsymbol{x}[k]-\boldsymbol{e}_{q} \Delta x\right)\right\},
\end{aligned}
$$

where $e_{q}$ is the vector with $q$-th entry equal to 1 and all other entries equal to $0 ; \Delta x$ is a small parameter $\left(\Delta x=1 \times 10^{-5}\right.$ was used in Sec. V). The right-hand side of (23) is obtained by numerical integration of the original system (2).

\section{REFERENCES}

[1] M. Zima, M. Larsson, P. Korba, C. Rehtanz, and G. Andersson, "Design aspects for wide-area monitoring and control systems," Proc. IEEE, vol. 93, no. 5, pp. 980-996, May 2005.

[2] Electric Power Research Institute, Report to NIST on the Smart Grid Interoperability Standards Roadmap (Contract No. SB1341-09-CN-0031), June 17, 2009.

[3] U.S.-Canada Power System Outage Task Force, ed. Final Report on the August 14, 2003 Blackout in the United States and Canada: Causes and Recommendations, April 2004.

[4] Y. Tamura and N. Yorino, "Possibility of auto- \& hetero-parametric resonances in power systems and their relationship with long-term dynamics," IEEE Trans. Power Syst., vol. PWRS-2, no. 4, pp. 890-896, November 1987.

[5] N. Yorino, H. Sasaki, Y. Tamura, and R. Yokoyama, "A generalized analysis method of auto-parametric resonances in power systems," IEEE Trans. Power Syst., vol. 4, no. 3, pp. 1057-1064, August 1989.

[6] I. Dobson, J. Zhang, S. Greene, H. Engdahl, and P. W. Sauer, "Is strong model resonance a precursor to power system oscillations?" IEEE Trans. Circuits Syst. I, vol. 48, no. 3, pp. 340-349, March 2001.

[7] E. H. Abed, M. A. Hassouneh, and M. S. Saad, "Instability monitoring and control of power systems," in Applied Mathematics for Reconstructed Electric Power Systems: Optimization, Control, and Computational Intelligence, J. H. Chow, F. F. Wu, and J. Momoh, Eds. New York: Springer, 2005, pp. 159-178.

[8] K. Wiesenfeld, "Noisy precursors of nonlinear instabilities," J. Statistical Phys., vol. 38, no. 5/6, pp. 1071-1097, 1985.

[9] I. Mezić, "Spectral properties of dynamical systems, model reduction and decompositions," Nonlinear Dyn., vol. 41, pp. 309-325, August 2005.

[10] C. W. Rowley, I. Mezić, S. Bagheri, P. Schlatter, and D. S. Henningson, "Spectral analysis of nonlinear flows," J. Fluid Mech., vol. 641, pp. 115-127, 2009.

[11] Y. Susuki and I. Mezić, "Nonlinear Koopman modes and coherency identification of coupled swing dynamics," IEEE Trans. Power Syst., vol. 26, no. 4, pp. 1894-1904, November 2011 (also, correction, this journal, vol. 26, no. 4, p. 2584, November 2011.).

[12] B. O. Koopman, "Hamiltonian systems and transformations in Hilbert space," Proc. National Academy of Sciences of the USA, vol. 17, no. 5, pp. 315-318, May 1931.

[13] K. Peterson, Ergodic Theory. Cambridge: Cambridge University Press, 1983.

[14] A. Lasota and M. C. Mackey, Chaos, Fractals, and Noise: Stochastic Aspects of Dynamics., 2nd ed. New York: Springer-Verlag, 1994.

[15] I. Mezić and A. Banaszuk, "Comparison of systems with complex behavior," Physica D, vol. 197, pp. 101-133, 2004.

[16] N. Wiener and A. Wintner, "Harmonic analysis and ergodic theory," American J. Math., vol. 63, no. 2, pp. 415-426, April 1941.

[17] Y. Susuki, I. Mezić, and T. Hikihara, "Global swing instability of multimachine power systems," in Proc. 47th IEEE Conference on Decision and Control, Cancun, Mexico, December 9-11, 2008, pp. 2487-2492.

[18] — "Coherent swing instability of power grids," J. Nonlinear Sci., vol. 21, no. 3, pp. 403-439, June 2011.

[19] B. Eisenhower and I. Mezić, "A mechanism for energy transfer leading to conformation change in networked nonlinear systems," in Proc. 46th IEEE Conference on Decision and Control, New Orleans, LA, December 12-14, 2007, pp. 3976-3981.

[20] P. Kundur, Power System Stability and Control. McGraw-Hill, 1994.

[21] IEEE/CIGRE Joint Task Force on Stability Terms and Definitions, "Definition and classification of power system stability," IEEE Trans. Power Syst., vol. 19, no. 2, pp. 1387-1401, May 2004.

[22] V. I. Arnold, Mathematical Methods of Classical Mechanics, 2nd ed. New York: Springer-Verlag, 1989.

[23] B. Avramović, P. V. Kokotović, J. R. Winkelman, and J. H. Chow, "Area decomposition for electromechanical models of power systems," Automatica, vol. 16, pp. 637-648, November 1980.

[24] J. R. Winkelman, J. H. Chow, B. C. Bowler, B. Avramović, and P. V. Kokotović, "An analysis of interarea dynamics of multi-machine systems," IEEE Trans. Power App. Syst., vol. PAS-100, no. 2, pp. 754 763, February 1981.

[25] S. Sastry and P. Varaiya, "Coherency for interconnected power systems," IEEE Trans. Automat. Contr., vol. AC-26, no. 1, pp. 218-226, February 1981.

[26] T. Athay, R. Podmore, and S. Virmani, "A practical method for the direct analysis of transient stability," IEEE Trans. Power App. Syst., vol. PAS-98, no. 2, pp. 573-584, March/April 1979. 
[27] M. A. Pai, Energy Function Analysis for Power System Stability. Kluwer Academic Pub., 1989

Yoshihiko Susuki (S'01-M'05) received the bachelor, master, and Ph.D. degrees in engineering from Kyoto University, Kyoto, Japan, in 2000, 2002, and 2005, respectively.

In 2005, he joined the Department of Electrical Engineering, Kyoto University, where he is currently a Lecturer. In 2008-2010, he was a Visiting Researcher in the Department of Mechanical Engineering, University of California, Santa Barbara, CA, under JSPS Postdoctoral Fellowship for Research Abroad. His research interests include power engineering, nonlinear dynamical systems, and control systems.

Igor Mezic (M'08) received the Ph.D. degree in applied mechanics from California Institute of Technology, Pasadena, CA, in 1994.

$\mathrm{He}$ is currently a Professor of the Department of Mechanical Engineering, and the Director of Center for Energy-Efficient Design and the Head of Buildings and Design Solutions Group of the Institute of Energy Efficiency at the University of California, Santa Barbara, CA. 